\title{
Religionen und Weltfrieden.
}

\section{Zum Friedens- und Konfliktlösungspotenzial von Religionsgemeinschaften. Internationales Symposium, 20. - 23. Oktober 2010, Osnabrück}

Können Religionen Frieden schaffen? Oftmals werden religiöse Gruppierungen lediglich als Eskalationsfaktoren und „Brandbeschleuniger“ in Gewaltkonflikten wahrgenommen. Worauf gründet also der Anspruch der verschiedenen Weltreligionen, Friedensmacht zu sein, und warum sieht die Wirklichkeit häufig anders aus? Unter welchen Bedingungen können Religionsgemeinschaften zu einer friedlichen Konfliktlösung beitragen und friedensstiftend wirken?

Einen Beitrag zur Klärung dieser Leitfragen möchte das vom 20. - 23. Oktober 2010 in Osnabrück stattfindende Symposium „Religionen und Weltfrieden. Zum Friedens- und Konfliktlösungspotenzial von Religionsgemeinschaften " leisten, welches von der Deutschen Stiftung Friedensforschung, dem Wissenschaftlichen Rat der Osnabrücker Friedensgespräche und dem Forschungsverbund Religion und Konflikt ausgerichtet wird. Die Veranstalter rücken hiermit gezielt das friedensfördernde und friedensstiftende Potenzial von Religionen und Religionsgemeinschaften in den Mittelpunkt, das bisher weder in der wissenschaftlichen Forschung noch in der öffentlichen Debatte eine hinreichende Berücksichtigung gefunden hat.

Renommierte Wissenschaftler und Moderatoren werden Fachkollegen sowie die interessierte Öffentlichkeit in ihren Vorträgen und Diskussionen zum Nach- und Weiterdenken anregen. Prof. Dr. Hans Küng, Gründer und Präsident der Stiftung Wel- tethos, eröffnet das Symposium mit dem Vortrag zum Thema „Eine gemeinsame Vision vom Frieden in der Welt? Religionen als treibende Kraft für eine Kultur des Friedens“. International bekannte Experten wie Mark Juergensmeyer, Scott Appleby u.a. setzen sich mit den Friedensbegriffen und dem friedensfördernden Selbstverständnis der fünf Weltreligionen Hinduismus, Buddhismus, Islam, Christentum und Judentum auseinander. Hier sind Antworten auf die Frage zu erwarten, wie Religionen dazu beitragen können, dass Konflikte gewaltlos ausgetragen und Friedensprozesse nachhaltig gefördert werden. Interdisziplinär forschende Persönlichkeiten wie David Little und Daniel Philpott sollen mit Hilfe aktueller Forschungsergebnisse aus Soziologie, Politik, Theologie und Recht neue Einsichten in den Themenkomplex Religion - Konflikt - Frieden geben. Einen weiteren Schwerpunkt setzt das Symposium durch Einblicke in die Praxis der religiösen Friedensarbeit in verschiedenen Konfliktregionen und entsprechenden Erfahrungsberichten von einschlägigen Akteuren wie Mohammed Abu-Nimer oder Pumla Gobodo-Madikizela.

Das vom Bundesministerium für Bildung und Forschung geförderte Symposium soll für nachhaltige Impulse in Wissenschaft und Praxis sorgen und so dazu veranlassen, künftig stärker auf die friedensfördernde Komponente von Religionen und Religionsgemeinschaften einzugehen.

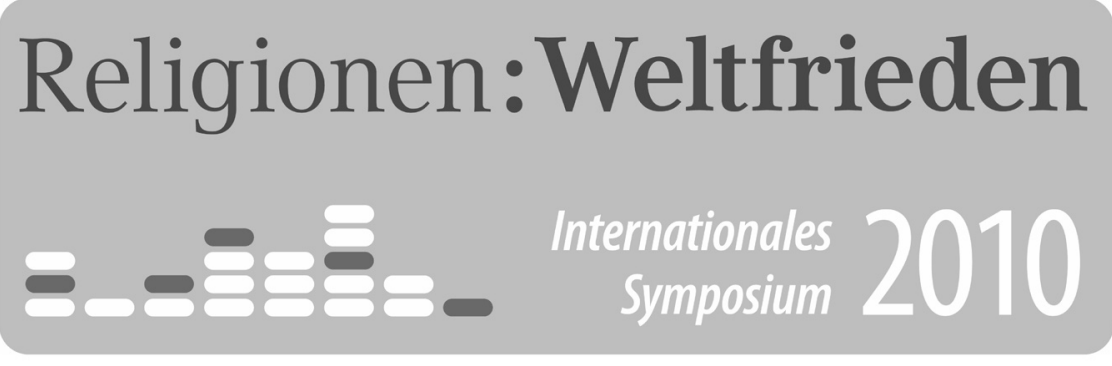

